\title{
Estratégias de preparação para o ENADE: contribuições para um curso de
}

\section{Odontologia}

\author{
Strategies of preparation for ENADE: contributions to a Dentistry course \\ Estrategias preparación para ENADE: aportaciones a un curso de Odontología
}

Recebido: 14/08/2021 | Revisado: 19/08/2021 | Aceito: 22/08/2021 | Publicado: 24/08/2021

\author{
Aluísio Ferreira Celestino Júnior \\ ORCID: https://orcid.org/0000-0002-1472-5155 \\ Centro Universitário do Estado do Pará, Brasil \\ E-mail: aluisio.junior@prof.cesupa.br \\ Patrícia Almeida Rodrigues \\ ORCID: https://orcid.org/0000-0003-3589-0771 \\ Centro Universitário do Estado do Pará, Brasil \\ E-mail: patrícia.almeida@prof.cesupa.br \\ Ceci Baker de Melo \\ ORCID: https://orcid.org/0000-0001-9827-3469 \\ Centro Universitário do Estado do Pará, Brasil \\ E-mail: ceci.melo@prof.cesupa.br \\ Viktória Karla Monteiro Cardoso \\ ORCID: https://orcid.org/0000-0002-3595-4758 \\ Centro Universitário do Estado do Pará, Brasil \\ E-mail: viktoriamonteiro@yahoo.com \\ Laryssa do Socorro dos Santos Pereira \\ ORCID: https://orcid.org/0000-0002-0051-7590 \\ Centro Universitário do Estado do Pará, Brasil \\ E-mail: loly1532@gmail.com \\ Adriana Viana Martins da Silva \\ ORCID: https://orcid.org/0000-0001-8087-2228 \\ Centro Universitário do Estado do Pará, Brasil \\ E-mail: adriana19900163@aluno.cesupa.br \\ Carolyne Oliveira Silveira \\ ORCID: https://orcid.org/0000-0002-4057-5867 \\ Centro Universitário do Estado do Pará, Brasil \\ E-mail: carolsilve@gmail.com \\ Elyne Mendes Faciola Xerfan \\ ORCID: https://orcid.org/0000-0001-8240-2019 \\ Centro Universitário do Estado do Pará, Brasil \\ E-mail: elyne_xerfan@hotmail.com \\ Lúcio Derek de Oliveira Ferreira \\ ORCID: https://orcid.org/0000-0003-0118-5249 \\ Centro Universitário do Estado do Pará, Brasil \\ E-mail: lucioferreira.odonto@ hotmail.com \\ Marina Lima Wandereley \\ ORCID: https://orcid.org/0000-0001-5246-039X \\ Centro Universitário do Estado do Pará, Brasil \\ E-mail: marina20900052@aluno.cesupa.br \\ Michel Wagner de Souza Matos \\ ORCID: https://orcid.org/0000-0002-0080-395X \\ Centro Universitário do Estado do Pará, Brasil \\ E-mail: michel21900141@ aluno.cesupa.br \\ Thayna Silva do Carmo Tavares \\ ORCID: https://orcid.org/0000-0002-1341-3096 \\ Centro Universitário do Estado do Pará, Brasil \\ E-mail: thaynasdocarmo@hotmail.com
}

\section{Resumo}

O presente trabalho apresenta o relato de experiência de intervenções pedagógicas voltadas para preparação ao Exame Nacional do Ensino Superior (ENADE) no âmbito do curso de Odontologia de uma faculdade no norte do Brasil. É apresentada neste artigo a contribuição específica de intervenções no contexto do eixo de Odontologia em Saúde Coletiva que compõem um Plano de Ação mais amplo com outras habilidade e competência do curso. A fase inicial se constituiu de pesquisa exploratória para identificação de potencialidades e fragilidades pertinentes aos temas do 
Componente Especifico do eixo e do Componente Geral que fazem intercessão com a Saúde Coletiva. Essas atividades propedêuticas foram construídas em duas fases, tendo em comum o uso de Metodologias Ativas de aprendizagem. A primeira (Componente Específico) foi predominantemente realizada através de Atividades Gamificadas e a segunda (Componente Geral) realizada através de debates mediados pela dinâmica conhecida como "Abrigo Subterrâneo" com modificações voltadas para aprofundamento de temáticas transversais, sendo sintetizada através da elaboração da ferramenta pedagógica denominada "Mapa Mental" construída pelos estudantes. Além de promover maior compreensão e interpretação das questões que compõem as temáticas dos componentes Geral e Específico, identificou-se, competências e/ou habilidades que necessitam ser mais bem trabalhadas e, consequentemente, desenvolvidas no contexto das disciplinas dos cursos e nas etapas subsequentes do Plano de Ação. Os resultados parciais descritos sugerem alternativas de intervenção para melhoria da aprendizagem discente, bem como possibilitar replicação das ações em outros contextos de formação.

Palavras-chave: Aprendizagem; Metodologias ativas; Odontologia; Ensino.

\begin{abstract}
This paper presents an experience report of pedagogical skills aimed at preparing for the National Higher Education Examination (ENADE) within the scope of the Dentistry course at a college in northern Brazil. In this article, the specific contribution of competences in the context of the Public Health Dentistry axis, which makes up a broader Action Plan with other skills and competences of the course, are emitted. The initial phase consists of exploratory research to identify strengths and weaknesses relevant to the themes of the Specific Component of the axis and the General Component that intersect with Public Health. These introductory activities were built in two phases, having in common the use of Active Learning Methodologies. The first (Specific Component) was predominantly carried out through Gamified Activities and the second (General Component) carried out through debates mediated by the Dynamics known as "Underground Shelter" with modifications aimed at deepening cross-cutting themes, being synthesized through the formation of the pedagogical tool called "Mental Map" constructed by the students. In addition to promoting greater understanding and interpretation of the issues that make up the themes of the General and Specific component, competences and/or skills that were classified as being better worked were identified and, consequently, developed in the context of the subjects of the courses and in the subsequent stages of the Plan of action. The partial results are incredible alternatives for intervention to improve student learning, as well as enabling the replication of actions in other training contexts.
\end{abstract}

Keywords: Learning; Active methodologies; Dentistry; Teaching.

\title{
Resumen
}

Este artículo presenta un relato de experiencia de competencias pedagógicas orientadas a la preparación para el Examen Nacional de Educación Superior (ENADE) en el ámbito del curso de Odontología en una facultad del norte de Brasil. En este artículo se emite el aporte específico de competencias en el contexto del eje Odontología en Salud Pública, que compone un Plan de Acción más amplio con otras habilidades y competencias del curso. La fase inicial consiste en una investigación exploratoria para identificar fortalezas y debilidades relevantes a los temas del Componente Específico del eje y el Componente General que se cruzan con Salud Pública. Estas actividades introductorias se construyeron en dos fases, teniendo en común el uso de Metodologías de Aprendizaje Activo. El primero (Componente Específico) se llevó a cabo predominantemente a través de Actividades Gamificadas y el segundo (Componente General) a través de debates mediados por la Dinámica conocida como "Refugio Subterráneo" con modificaciones encaminadas a profundizar temas transversales, sintetizándose a través de la formación de la herramienta pedagógica denominada "Mapa mental" construida por los estudiantes. Además de promover una mayor comprensión e interpretación de los temas que componen las temáticas del componente General y Específico, se identificaron competencias y / o habilidades que fueron calificadas como mejor trabajadas y, en consecuencia, desarrolladas en el contexto de las asignaturas de los cursos y en las etapas posteriores del Plan de acción. Los resultados parciales son increíbles alternativas de intervención para mejorar el aprendizaje de los estudiantes, además de posibilitar la replicación de acciones en otros contextos formativos.

Palabras clave: Aprendizaje; Metodologías activas; Odontología; Enseñanza.

\section{Introdução}

No Brasil, o processo de ensino e aprendizagem é baseado, tradicionalmente, na teoria da transmissibilidade, ou seja, na transmissão de informações. Também conhecido como ensino tradicional. Esta proposta de aprendizagem é baseada "no transmitir", utilizando-se, para tanto, de aulas expositivas, nas quais os estudantes aprendem de forma passiva as informações transmitidas pelo professor (Couto, 2019).

$\mathrm{Na}$ área da saúde, predominantemente, estão sendo adotadas formas diferenciadas de ensino-aprendizagem e de 
organização curricular na perspectiva de integrar a teoria com a prática e o ensino com o serviço, além de desenvolver capacidade reflexiva acerca de problemas reais, assim como, planejamento de ações originais e criativas capazes de modificar a realidade social (Reul, 2016).

Dessa forma, a introdução de metodologias ativas associada a gamificação seria uma estratégia de ensino diferenciada para promover uma aprendizagem colaborativa e significativa para estudantes da área de saúde (Morano, 2021).

As metodologias ativas permitem também a articulação entre a universidade, o serviço e a comunidade, por possibilitar uma leitura e intervenção consistente sobre a realidade, valorizar todos os atores no processo de construção coletiva e seus diferentes conhecimentos e promover a liberdade no processo de pensar e no trabalho em equipe (Caldarelli, 2017).

Para que essa mudança seja realmente vivenciada, a abordagem de ensino-aprendizagem necessita incluir metodologias participativas, que estimulem o papel ativo dos estudantes e processos avaliativos processuais e formativos. Isso ganha maior sustento quando estudantes de Odontologia reconhecem que as Metodologias Ativas de aprendizagem favorecem a capacidade de aprender de forma autônoma, desenvolvendo o pensamento crítico, com a finalidade de analisar soluções de problemas e refletir sobre elas [...] (De-Carli et al, 2019).

À vista disso, este trabalho destina-se a relatar a experiência de intervenções pedagógicas voltadas para preparação de alunos de graduação para o Exame Nacional do Ensino Superior (ENADE) no âmbito do curso de odontologia de uma faculdade no norte do Brasil nas quais foram utilizadas metodologias ativas, parte delas com ferramentas de gamificação com o intuito de dinamizar o processo ensino/aprendizagem dos discentes e assim, a partir dos resultados obtidos através deste estudo possibilitar a replicação dessas ações em outros contextos de formação.

\section{Metodologia}

A estratégia de ação foi concebida e desenvolvida por docentes e mestrandos do Programa de Mestrado Profissional em Clínica Odontológica e voltadas para uma turma do sétimo período do curso de graduação de odontologia formada por 37 estudantes que ao final do curso se submeterão ao Exame Nacional de Desempenho do Estudante-ENADE.

Aqui são descritas as intervenções pertinentes ao contexto do eixo da Saúde Bucal Coletiva as quais compõem um Plano de Ação mais amplo com outras habilidade e competência do curso.

Esta estratégia metodológica se desenvolveu em duas linhas centrais. A primeira delas abordando o Componente Geral que constitui o Exame Nacional de Desempenho do Estudante e a segunda abordando o Componente Específico. O Componente Geral versa sobre temas abrangentes contemplando diversas áreas da atividade humana tais como economia, política, cultura, sustentabilidade, comunicação, violência, demografia, saúde, políticas públicas, atualidades, populações vulneráveis etc. O Componente Específico refere-se as diversas temáticas que compõe as habilidades e competências especificas previstas nas diretrizes curriculares dos cursos de graduação em odontologia no Brasil.

Nesta estratégia, os autores, definiram como tema do Componente Específico da odontologia a Política Nacional de Saúde Bucal, tema ligado ao eixo de Saúde Bucal Coletiva. Os temas relacionados ao Componente Geral foram trabalhados a partir de dinâmica conhecida como "Abrigo subterrâneo" descrito adiante, recurso útil para eliciar reflexão sobre diversos temas que compõem também o Componente Geral.

No Componente Específico da odontologia, o tema trabalhado foi desenvolvido de maneira híbrida, parte remotamente, via Google Classroom (estudo prévio) e parte presencialmente. O estudo prévio individual foi feito através de leitura de texto oficial do Ministério da Saúde (Brasil, 2004) sobre a Política Nacional de Saúde Bucal e um vídeo curto sobre este tema disponível na Plataforma Youtube disponibilizado pelo Ministério da Saúde.

Um grande desafio para os docentes e mestrandos seria a "Garantia de Preparo", pois nenhum deles ministrava aulas 
aos 37 estudantes de graduação envolvidos na ação, sendo este um trabalho de interesse voluntário para os graduandos. Não havia pontuação, por exemplo, que os “motivasse” à participação.

Como estratégias de motivação foram realizadas comunicações pela Plataforma Google Classroom bem como visita aos alunos, expondo a estratégia e estimulando-os a participação, destacando as dinâmicas de grupo gamificadas e premiações (brindes) envolvidas em uma gincana de conhecimentos.

Depois de enviado o material aos estudantes, a estratégia presencial foi desenvolvida inicialmente através de um Quiz utilizando a ferramenta Socrative no recurso conhecido como Corrida Espacial.

Tomando como base o desempenho dos estudantes ao longo do curso, os grupos foram divididos em equipes com desempenho variado para evitar desequilíbrio entre as equipes. Esta informação, entretanto, estava restrita aos facilitadores da dinâmica (docentes e mestrandos). Cada um dos sete times foi nomeado por cores.

Cada time recebia em seu próprio (um por grupo) Smarthphone um código de acesso à plataforma Socrative com um Quiz de 15 perguntas acerca do tema específico. À medida que assinalava a questão na plataforma, sua resposta, representada por um foguete com a cor de sua equipe, aparecia na tela projetada na sala de Metodologias Ativas da Instituição. A cada resposta correta o foguete progredia. A estratégia de visualizar a progressão do foguete e o tempo previamente delimitado para a atividade estimulavam a competição entre as equipes.

Os itens (questões) e suas respostas eram simultaneamente acompanhadas pelos facilitadores para identificar dificuldades por parte dos grupos, bem como o percentual de aproveitamento geral e específico em cada item do Quiz. Estes itens foram elaborados no sentido de estimular a compreensão da política ao mesmo tempo que instigasse a reflexão. Essa estratégia, em geral, gera eventuais dúvidas que adiante, no feedback de cada item, era devidamente orientado pelos facilitadores.

Entre as estratégias de motivação à participação, cada estudante recebeu um brinde relacionado a biossegurança (EPIs). Os melhores times também recebiam outros prêmios de acordo com seu desempenho: primeiro, segundo e terceiros lugares na atividade gamificada.

Como atividade de desempate estava previsto o lançamento de perguntas orais na dinâmica de "toca a sineta" que prevê uma pergunta e em seguida a resposta por alguém que primeiramente aciona a sineta. Nesta atividade, dois times empataram em segundo lugar e concorreram com novas perguntas para esta etapa de pontuação. Todas com feedback imediato.

Definidos os vencedores, foi realizada a "cerimônia de premiação" dos times vencedores e em seguida foi servido um lanche coletivo. Todos os brindes e lanches foram conseguidos através de patrocínio mediado pelos facilitadores.

As atividades realizadas foram fotografadas e postadas em redes sociais oficiais da instituição, valorizando o trabalho desenvolvido, bem como dando visibilidade aos patrocinadores das ações que ofereceram materiais odontológicos diversos e que foram entregues aos times vencedores.

O Componente Geral foi desenvolvido em dia distinto da atividade anterior. Utilizou-se a estratégia conhecida como “Abrigo Subterrâneo” (Secchi, \& Feijó, 2015), agregando outros recursos para consolidação da estratégia.

\section{"Abrigo subterrâneo":}

Cada estudante deveria selecionar 7 pessoas de uma relação de 15 personagens/situações fictícias que envolvem as temáticas centrais de Componente Geral. Dentre os quinze personagens relacionados, cada participante deveria escolher apenas sete que seriam salvos de uma hecatombe, protegendo-se em um abrigo subterrâneo. Cada personagem da dinâmica representa valores e situações diferentes que se reportam direta ou indiretamente aos diversos temas que comumente fazem parte do Componente Geral da ENADE. 
Atividade individual $\rightarrow$ Desenvolvida entre 10 e 13 minutos. Nela cada estudantes escolhe os seus sete personagens/situações assinalando em uma folha de papel A4.

Atividade Grupal $\rightarrow$ Desenvolvida até 30 minutos (grupo de cinco membros). Nesta etapa, cada estudante confronta sua escolha com a de mais quatro colegas (equipes de cinco, portanto) e definem apenas sete personagens/situações para o grupo. Nesta etapa, a reflexão é espontaneamente maior por conta de valores, conhecimento e histórias de cada pessoas no grupo.

ANÁLISE PRELIMINAR (Feita com auxílio da equipe de facilitadores);

No quadro branco da sala são colocadas as opções relacionadas de cada time. Todos os participantes são instigados a verificar o que convergiu e o que divergiu entre os times apresentados no quadro branco.

Momento 1: Análise de convergência

Considerando a seleção de todos os grupos, são destacadas as personagens/situações excluídas por todos os times, colocando também no quadro. Nesta etapa, os facilitadores verificam os personagens e perguntam aos participantes qual a razão de exclusão daquele personagem. A discussão deve aprofundar as razões desta exclusão. Quando estas razões não ficam claras ou não são expostas pelos participantes, são mencionadas pelos facilitadores para aprofundamento.

Considerando a seleção de todos os times, são também destacadas as personagens/situações incluídas por todos. Colocando também no quadro.

Conduzida por um dos facilitadores, nova reflexão (breve) é feita para explorar as vertentes importantes que conduziram a escolha daquele personagem/situação por todos os grupos.

\section{Momento 2: Análise de confronto}

O facilitador franqueia a palavra para que cada time pergunte a outros times quais as razões de suas escolhas considerando situações específicas.

Obs.: Em geral não há necessidade de instigar o debate, pois são situações de limite/fronteira e cotidianas que favorecem a discussão, mas há que se considerar e planejar eventuais motivações para grupos menos participativos. Ex.: "Por que vocês incluíram personagem 3, deixando o personagem 9 fora do abrigo?" ou ainda "Restará harmonia no grupo no abrigo se mantiverem o personagem 7 e o personagem 11 juntos?”

Uma reflexão breve deve ser conduzida por um dos facilitadores para evoluírem para fase seguinte.

\section{Momento 3: uma síntese possível}

Cada grupo recebeu por sorteio um dos personagens/situação sobre o qual deveria fazer um "Mapa Mental” e apresentá-lo aos demais grupos. O Mapa Mental deveria ser construído com o máximo de variáveis sobre os temas que cada personagem/situação representa. Foi explicado que cada equipe teria a liberdade de falar sobre todas as variáveis (quando forem poucas) ou sobre as principais (quando forem muitas). O tempo de cinco minutos para cada grupo é a melhor referência para a exposição dos grupos. Ao final, seriam selecionados os três melhores Mapas Mentais.

A seleção dos melhores trabalhos é feita por alguns dos facilitadores que deveriam tomar como referência a proficiência evocada pelo personagem/situação e exposta no Mapa Mental e os temas a ele subjacentes. O grau de profundidade da argumentação é importante quesito a ser avaliado, bem como eventuais lacunas que o tema central evoca não contemplada pelo grupo. 
As Estratégias de Motivação do segundo dia foram semelhantes a etapa anterior: premiação por participação (brindes, em geral EPIs), além de certificado emitido pela coordenação de curso. A premiação por desempenho nesta etapa foi restrita ao desempenho por time, não havendo premiação por desempenho Individual.

Estas estratégias compõem, entretanto, um conjunto de ações que contemplam conteúdos de outras áreas da odontologia e que deverão ser aplicadas no decorrer de 18 meses de preparação paralela, tempo este que antecede a aplicação do Exame Nacional de Desempenho do Estudante.

\section{Resultados e Discussão}

O perfil do egresso do curso de odontologia previsto nas Diretrizes Curriculares Nacionais (Brasil, 2021) publicadas em junho de 2021, consolidam as características desafiadoras da formação deste profissional. Logo em seu inciso I prevê um profissional" generalista, dotado de sólida fundamentação técnico-científica e ativo na construção permanente de seu conhecimento". Estratégias que corroboram com este perfil devem ser constantemente implementadas, revisadas, a\Atualizadas. A proposta complementar de formação desenvolvidas pelos autores estão em consonância com este perfil, avançando em outras características que uma formação consistente exige.

Na proposta metodológica desenvolvida também foram contempladas outras características necessárias ao cirurgião dentista. "humanístico e ético, atento à dignidade da pessoa humana e às necessidades individuais e coletivas, promotor da saúde integral e transformador da realidade em benefício da sociedade" descrito no inciso II do art. 30. Tal reflexão proporcionada pela dinâmica do Abrigo Subterrâneo tem no contexto da Saúde Bucal Coletiva um campo bastante conectado com este perfil. Esta dinâmica permitiu estabelecer uma relação entre os valores individuais e coletivos encontrados em personagens/situações fictícias ligadas ao contexto de atuação profissional.

O trabalho foi realizado com estudantes de graduação cuja atividade não é obrigatória, pois os facilitadores não são seus docentes, portanto, os desafios de convencimento à participação em situações semelhantes, são maiores. Autores como Nascimento, Kosminsky e Cavalcanti (2018) encontraram um grau importante de desmotivação de estudantes de odontologia em um grupo que sinalizou que suas principais dificuldades estavam relacionadas a atitudes de alguns docentes em relação aos discentes, falhas na metodologia de ensino, bem como inapropriadas instalações físicas da unidade de ensino, além da matriz curricular.

Em que pese um conjunto de metodologias estarem disponíveis para serem utilizadas na aprendizagem ativa, há ainda um passo importante a ser dado pelo conjunto majoritário de professores que as utilizam com proficiência (Maciel et al, 2019). Ao adotar estratégias metodológicas ativas como a sugerida nesta estratégia, a adesão é certamente maior. Mas os elementos de motivação presentes nas ações não seriam tão relevantes se o alcance do aprendizado não for a meta primária a ser alcançada.

Os recursos utilizados na estratégia proposta têm grande apelo pedagógico ao inserir estudantes de mestrado no contexto de formação da graduação. A utilização de Tecnologias de Informação e Comunicação como as que foram utilizadas nas duas etapas da atividade passam a ser percebidas como recursos preciosos no agir pedagógico. Mediar o processo de aprendizagem com estes recursos compõem atualmente um conjunto de ferramentas essenciais que o docente não deve prescindir (Brito, 2019) e que precisa ser apropriado pelo mestrando, potencial docente.

Dentre os recursos da Aprendizagem Ativa há que se preocupar com elementos importantes como a Garantia de Preparo, por exemplo (Oliveira et al, 2018) como aquela etapa obrigatória na estratégia de Team Based Learning-TBL (Souza, 2020). Embora a turma do sétimo período já tenha visto a temática proposta no início do curso, parte dos estudantes possivelmente não lembravam dos conteúdos trabalhados. A leitura de texto específico é sempre mais desafiadora sem uma motivação maior. O vídeo, por sua vez, tem menor resistência, no entanto, nem todos os estudantes assistiram o vídeo. Dos que 
leram o texto e assistiram o vídeo, parte se deveu a atividade de gincana que previa premiação.

Atualmente há muitas alternativas de "Garantia de Preparo" proporcionada por plataformas digitais. Estes recursos são cada vez mais valorizados na prática docente e de grande aceitação pelos discentes. Muitos aplicativos da odontologia produzidos para estas plataformas auxiliam na retenção de conteúdos e no engajamento (Celestino Júnior et al. 2018). Há necessidade, entretanto, que estejam conectados com a ideia central que é objeto do aprendizado.

Outro aspecto relevante que contribui para a estratégia das atividades gamificadas escolhida é que, além do reflexo positivo relacionado a motivação, ela se destaca também pela melhor retenção de conhecimentos abordados por essa metodologia (Oliveira et al, 2021).

Corrobora com esta característica, o feedback imediato exigido nas técnicas de aprendizagem gamificadas. Nas estratégias realizadas, cada etapa tinha 'devolutiva imediata' o que favorece o maior interesse em compreender o conteúdo no momento em que eventuais dúvidas acontecem. É importante destacar também que todas as etapas desenvolvidas tinham suas próprias regras que eram repassadas na forma de instruções claras e se certificando de que havia compreensão das normas de cada etapa, seus objetivos, procedimentos e recompensa (Fardo, 2013; Burke, 2015). Clareza nas instruções é uma exigência de qualquer atividade pedagógica proposta aos estudantes. Em atividades gamificadas não seguir esta recomendação é correr sérios riscos de conflitos durante sua execução.

A dinâmica do "Abrigo Subterrâneo" pode ser utilizada em vários contextos, alguns deles que envolvem características de liderança, autonomia, solidariedade, humanização, alteridade, respeito ao outro, num contexto que envolve diversos contextos humanos. O exercício de reflexão fomentou os temas transversais exigidos comumente nos componentes gerais de concursos tal como no ENADE, mas exigia também o confronto de valores que envolvem a visão de ser humano, de profissional e de sociedade que se almeja tal como propõem em seu inciso II do artigo 30 das diretrizes Curriculares Nacionais do curso de odontologia, qual seja um perfil " humanístico e ético, atento à dignidade da pessoa humana e às necessidades individuais e coletivas, promotor da saúde integral e transformador da realidade em benefício da sociedade; (BRASIL, 2021).

O recurso complementar da técnica do Mapa Mental permitiu não somente sintetizar as ideias gerais de cada grupo, mas tomar posição sobre uma ideia e ao que ela está subjacente, ou seja, buscar e destacar as razões que sustentam uma argumentação. Paralelamente, este recurso permite além da síntese, uma forma de retenção melhor de conteúdos complexos em um formato que privilegia a memória visual e que propicia, de maneira secundária, o aprofundamento, a análise (Galante, 2020; Carabetta Júnior, 2013). Esta técnica, no contexto desenvolvido, pelos autores previa apresentação oral, valorizando, portanto, outra dimensão de competência do cirurgião dentista, a comunicação.

O desafio de fomentar a responsabilidade com o próprio conhecimento não é pequeno. Embora em grupos tão heterogêneos como os que comumente se encontra em uma turma de graduação, pareçam ser barreiras para alcançar maior engajamento e maturidade com a formação, há alternativas de boa aceitação e de resultados mais sólidos que precisam ser estimulados. A estratégia desenvolvida tem caráter complementar e, em geral, este tipo de metodologia estimula a participação nos grupos, onde há grande troca de conhecimento entre os pares. Essa pluralidade quando bem conduzida enriquece o grupo com as habilidades e competências exigidas do profissional mais adiante. Motivação, engajamento e retenção dessas habilidades não são auferidas por uma estratégia isolada, mas por um contexto geral onde essa autonomia e integração venham a ser trabalhados com métodos adequados.

\section{Considerações Finais}

O campo de formação da Saúde Bucal como outros campos da área da saúde, vêm sofrendo amplas transformações, parte delas relacionadas às mudanças exigidas no contexto contemporâneo em que habilidades não inteiramente novas são 
exigidas do profissional. Em que pese, este campo profissional exigir estas transformações, ou seja, novas adaptações e abordagem, há um descompasso que precisa ser reduzido. Parte destas transformações são trabalhados com recursos metodológicos que a área da saúde abraçou em primeira mão: as metodologias de Aprendizagem Ativa. A autonomia do estudante na busca do conhecimento é cada vez mais estimulada por estes recursos, o engajamento, a participação. Embora a técnica seja importante no contexto geral dessa nova forma de aprendizagem, é necessário vincular esse conjunto de ferramentas aos valores importantes de indivíduo e coletividade valores humanos, valores da sociedade, ética. Que profissional as instituições estão formando? É uma pergunta que extrapola o a dimensão cognitiva, tecnicista. Aliar estratégias metodológicos a partir dessa premissa parece ser um bom caminho para um perfil de egresso que tanto nossas diretrizes curriculares nacionais pretendem alcançar.

Isto posto, há que se pensar que a educação não se exaure nestes tipos de ações e atividades realizadas isoladamente, faz-se necessário ampliar o espectro que a comunidade acadêmica vem contemporaneamente exigindo e que, ávida, necessita. A aprendizagem progressivamente exige de seus atores, inovação e engajamento com consistência. A partir dos resultados obtidos, novas ações e habilidades são requeridas. Na proposta, inicialmente, implementada fica claro que outras áreas são complementares além do Componente Geral e das Competências relacionadas ao eixo de Saúde Bucal Coletiva. O desafio que se impõe é integrar esses saberes e dos outros campos da odontologia, no local onde ele se faz mais necessário, qual seja, na mente reflexiva desses atores em formação. Esse processo, sem uma avaliação processual feita por todos os envolvidos, seria apenas um conjunto de tarefas. Com a avaliação, retifica-se os caminhos metodológicos mais exitosos, bem como se identifica eventuais fragilidades, aquelas nas quais as mudanças de rumo poderão trazer resultados mais duradouros quando regularmente empreendidos. O processo de aprendizagem isolado, com foco único perde em efetividade e ao invés de consolidar, dilui-se. Este é o novo desafio, de fato desafio constante a ser construído, pois o ensino é sempre reconstrução, a fronteira do conhecimento está continuamente sendo superada, metodologias centradas no protagonismo dos estudantes, seguramente tem maiores chances de prosperar.

\section{Referências}

Barreto, C., Becker, E., \& Ghisleni, T. (2019). Gamificação: uma Prática da Educação 3.0. Research, Society and development, 8(4), 1-21, 2019. https://rsd.unifei.edu.br/index.php/rsd/article/view/942/751.

Brasil. (2004). Diretrizes da Política Nacional de Saúde Bucal. Coordenação Geral de Saúde Bucal. http://conselho.saude.gov.br/web_comissoes/cisb/doc/politica_nacional.pdf

Brasil. Ministério da Educação/Conselho Nacional de Educação. (2021). Diretrizes Curriculares Nacionais do curso de graduação em Odontologia. Resolução nº 3, de 21 de junho de 2021. https://www.in.gov.br/web/dou/-/resolucao-n-3-de-21-de-junho-de-2021-327321299.

Brito, J. (2019). O Uso das Tecnologias da Informação e Comunicação (TICs) como Instrumento Mediador no Processo de Ensino-Aprendizagem. (2019). 77f. (Monografia) - Universidade Federal Rural do Semi-árido, Rio Grande do Norte,

Burke, B. (2015). Gamificar: como a gamificação motiva as pessoas a fazerem coisas extraordinárias. DVS editora.

Carabetta Júnior, V. (2013) A utilização de mapas conceituais como recurso didático para a construção e interrelação de conceitos. Revista Brasileira de Educação Médica, 37(3), 441-447, 2013.

Carvalho, W., Cawahisa, P., Scheibel, P., Botelho, J., Terada, R., Rocha, N., da Lolli, L., \& Fujimaki, M. (2016). Aceitação da utilização de metodologias ativas nos estágios no SUS por discentes da graduação e pós-graduação em Odontologia. Revista da ABENO, 16(1), 88-98.

Celestino Júnior, A., Cruz, J., \& Santos, Y., (2018). Aplicativos Móveis na Odontologia: Uma Revisão Integrativa. REAS, Revista Eletrônica Acervo Saúde. Vol. Sup. 14, S1819-S1924.

Fardo, M., (2013). A gamificação aplicada em ambientes de aprendizagem. Novas Tecnologias na Educação. CINTED-UFRGS: 11(1), http://www.seer.ufrgs.br/renote/article/download/41629/26409.

Galante, C., (2020) O uso de mapas conceituais e de mapas mentais como ferramentas pedagógicas no contexto educacional do ensino superior. Revista Eletrônica Múltiplo Saber, 23, 1-23, 2014. 
Research, Society and Development, v. 10, n. 11, e88101119432, 2021

(CC BY 4.0) | ISSN 2525-3409 | DOI: http://dx.doi.org/10.33448/rsd-v10i11.19432

Maciel, S., Silva, K., Melo, J., \& Soares, D., (2019). Metodologia Ativa aplicada ao Ensino odontológico um panorama Nacional a partir de um estudo Bibliométrico. Arch Health Invest 8(1).

Maranhão, K., \& Reis, A., (2019). Recursos de gamificação e materiais manipulativos como proposta de metodologia ativa para motivação e aprendizagem no curso de graduação em odontologia. Rev. Bra. Edu. Saúde, 9(3), 1-07.

Morano, D., (2021). Convergência entre gamificação e metodologias ativas: ferramentas no ensino de anatomia humana. 2021. 90 f. Dissertação (Mestrado em Ciências Morfofuncionais) - Faculdade de Medicina, Universidade Federal do Ceará, Fortaleza, 2021.

Nascimento, M., Kosminsky, M. \& Cavalcanti, S., (2018). Desmotivação entre estudantes de Odontologia: uma análise qualitativa. Revista da ABENO, 18(4):112-119. 10.30979/rev.abeno.v18i4.608.

Oliveira, A., Rambo, A., Gonçalves, L., Bosso, J., \& Haas, P. (2021). Efetividade do uso da gamificação na educação em saúde. Revista científica multidisciplinar. 2(6), e26422.

Oliveira, B., et al. (2018). Team-Based Learning como Forma de Aprendizagem Colaborativa e Sala de Aula Invertida com Centralidade nos Estudantes no Processo Ensino-Aprendizagem. Revista brasileira de educação médica 42 (4), 86-95; 2018.

Reul, M., Lima, E., Irineu, K., Lucas, R., Costa, E., \& Madruga, R. (2016). Metodologias ativas de ensino aprendizagem na graduação em Odontologia e a contribuição da monitoria - relato de experiência. Revista da ABENO, 16(2), 62-68.

Secchi, L., \& Feijó, J. (2015). Efeitos da urgência sobre o nível de participação em processos deliberativos. RACE, Revista de Administração, Contabilidade e Economia, 14 (3), 905-924. http://editora.unoesc.edu.br/ index.php/race.

Souza, J., Macedo, T., \& Coelho, H., (2021). Projeto Englishtalks: Uma proposta de ensino e aprendizagem da Língua Inglesa mediada pelo Google Meet e portal even3 em tempos de pandemia Research, Society and Development, 10(7), e26010716068.

Sousa, C. (2020). Metodologias ativas de ensino aprendizagem na área da saúde: revisão de literatura. Revista da faculdade facit. 21(1), 51-62. https://jnt1.websiteseguro.com/index.php/JNT/article/download/767/561. 